\title{
A New Design Method for Two-Channel Perfect Reconstruction IIR Filter Banks
}

\author{
S. C. Chan, J. S. Mao, and K. L. Ho
}

\begin{abstract}
In this paper, a new method for designing perfect reconstruction (PR) two-channel causal stable IIR filter banks is introduced. It is based on a structure previously proposed by Phoong et al. [2]. By using a combination of allpass and linear-phase FIR functions, the bumping problem found in the conventional structural PR filter bank is significantly suppressed. The design problem is formulated as a polynomial approximation problem and is solved effectively using the Remez exchange algorithm. Filter banks with flexible stopband attenuation and system delay can readily be obtained using the proposed algorithm.
\end{abstract}

Index Terms-Design method, filter banks, IIR filters, perfect reconstruction.

\section{INTRODUCTION}

$\mathbf{P}$ ERFECT reconstruction (PR) IIR filter banks are very attractive because of their potentially low system delay and better frequency responses compared with their FIR counterpart [1]. The two-channel IIR filter bank reported in [2] is particularly useful because of its simple design procedure and good performance. It is parameterized by two functions $\alpha(z)$ and $\beta(z)$, which can be chosen as an allpass function to obtain filterbanks with very high stopband attenuation. Unfortunately, a bump of about $4 \mathrm{~dB}$ always exists near the transition band of the analysis and synthesis filters. Moreover, the stopband attenuation of the analysis highpass filter is about $10 \mathrm{~dB}$ lower than that of the lowpass filter. To overcome these problems, an improved algorithm using general rational functions for $\alpha(z)$ and $\beta(z)$, instead of the allpass function, was recently proposed in [4]. The bumping problem in the transition band is considerably suppressed, but the design procedure is considerably more complicated and the stability of the IIR filters cannot be guaranteed. In this paper, $\beta(z)$ and $\alpha(z)$ are chosen as allpass and linear-phase FIR functions, respectively. One of the advantages is that the high stopband attenuation, low implementation complexity, and low coefficient sensitivity of the allpass function are preserved. Moreover, the bumping can still be suppressed with the use of a linear-phase FIR function. This has previously been observed in [5], but no design procedure was given. In this paper, we show that the design of such FIR functions can be formulated as a polynomial approximation problem, which can be solved readily using existing techniques. The new method also

Manuscript received December 17, 1999. The associate editor coordinating the review of this manuscript and approving it for publication was Prof. A. M Sayeed.

The authors are with the Department of Electrical and Electronic Engineering, The University of Hong Kong, Hong Kong (e-mail: scchan@eee.hku.hk; jsmao@eee.hku.hk; klho@eee.hku.hk).

Publisher Item Identifier S 1070-9908(00)06518-4. offers considerable freedom in controlling the stopband attenuation and delay of the highpass filters.

\section{THE PROPOSED METHOD}

Consider a two-channel critically decimated multirate filter bank, as shown in Fig. 1(a). The aliasing is eliminated if the synthesis filters are chosen as $G_{0}(z)=-H_{1}(-z)$ and $G_{1}(z)=H_{0}(-z)$, where $H_{0}(z)$ and $H_{1}(z)$ are, respectively, the low-pass and high-pass analysis filters. In [2], the analysis filters are chosen as

$$
H_{0}(z)=\frac{\left(z^{-2 N}+z^{-1} \beta\left(z^{2}\right)\right)}{2}
$$

and

$$
H_{1}(z)=-\alpha\left(z^{2}\right) H_{0}(z)+z^{-2 M-1}
$$

where $\alpha(z)$ and $\beta(z)$ are identical and the delay $M$ is equal to $2 N-1$. It is interesting to note that the system is PR for any choices of $\alpha(z)$ and $\beta(z)$. Because of (2.1), $\beta(z)$ can be chosen as a polynomial or a rational function. In [2], IIR filter banks are obtained by choosing $\beta(z)$ as a causal stable allpass function.

In this paper, $\beta(z)$ and $\alpha(z)$ are chosen as allpass and linearphase FIR functions, respectively, to realize PR filter banks with approximately linear phase. Let's assume that $\beta(z)$ is properly designed so that $H_{0}(z)$ is an approximately linear-phase lowpass filter with reasonably good frequency response, i.e., $H_{0}(z) \approx e^{-j 2 N \omega}$ in its passband and zero elsewhere. The design of such an allpass-based lowpass filter has previously been addressed in [3], where the problem is formulated as an eigenvalue problem. From (2.1), it can be seen that the frequency response of $H_{1}(z)$ depends on $H_{0}(z)$ and $\alpha(z)$. Let $\omega_{p}$ and $\omega_{s}$ be the passband and stopband cutoff frequencies of $H_{1}(z)$, respectively. The error function $E(\omega)$ of $H_{1}(z)$ is

$$
E(\omega)=e^{-j \omega(2 M+1)}-\alpha\left(e^{2 j \omega}\right) H_{0}\left(e^{j \omega}\right)-H_{d}\left(e^{j \omega}\right)
$$

where $H_{d}\left(e^{j \omega}\right)$ is the desired response and is equal to $e^{-j \omega(2 M+1)}$ for $\omega_{p} \leq \omega \leq \pi$ and zero otherwise. Since the minimization in (2.2) involves $\alpha\left(e^{2 j \omega}\right)$, which is periodic with period $\pi$, it is different from the conventional Chebyshev approximation. In fact, for a given value of $\omega \in[0, \pi / 2], \alpha\left(e^{2 j \omega}\right)$ will affect the values of $H_{1}\left(e^{j \omega}\right)$ at $\omega$ as well as $\omega+\pi$. If $H_{0}(z)$ is a reasonably good lowpass filter with $H_{0}(z) \approx e^{-j 2 N \omega}$ in its passband, then $\alpha\left(e^{2 j \omega}\right)$ should approximate $e^{-j(2 M+1-2 N) \omega}$ for $\omega \in[0, \pi / 2]$. If $\alpha(z)$ is a type-II FIR function, its magnitude should be approximately equal to one, except around $\omega=\pi / 2$, where it decreases to zero. It then follows from (2.1) that the passband ripples of $H_{1}\left(e^{j \omega}\right)$ is approximately equal to 


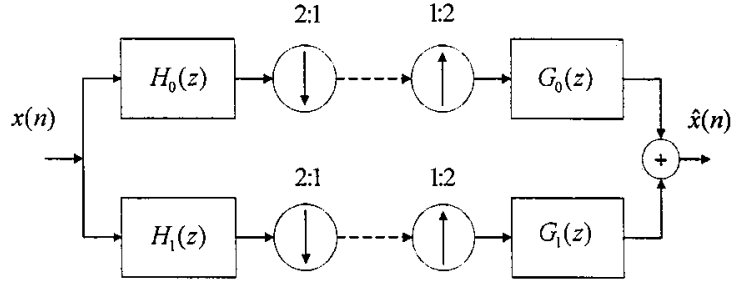

(a)

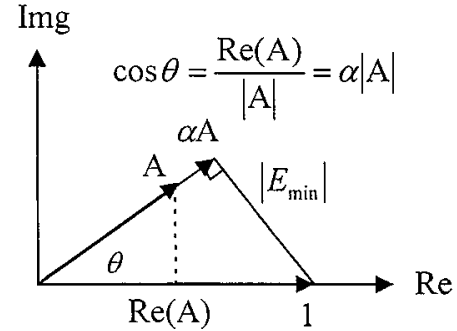

(b)

Fig. 1. (a) Two-channel multirate filter bank. (b) Geometric interpretation of (2.5).

the stopband error of $H_{0}\left(e^{j \omega}\right)$. This allows us to minimize only the stopband attenuation of $H_{1}\left(e^{j \omega}\right)$ using $\alpha\left(e^{2 j \omega}\right)$, instead of minimizing (2.2) over the pass- and stopbands, and relies on the high stopband attenuation of $H_{0}\left(e^{j \omega}\right)$ to achieve a small passband ripples. By increasing the length of $\alpha(z)$, the stopband attenuation of $H_{1}\left(e^{j \omega}\right)$ can be improved in exchange for a greater delay. In fact, the total system delay is given by $2 N+2 M+1$. Let $\alpha(z)$ be a Type-II linear-phase FIR filter given by

$$
\begin{aligned}
\alpha\left(e^{j \omega}\right) & =e^{-j \omega M_{o d d} / 2} \cos (\omega / 2) P(\cos \omega), \\
P(\cos \omega) & =\sum_{k=0}^{L} a_{k}(\cos \omega)^{k} .
\end{aligned}
$$

From (2.1) and (2.3), it can be seen that $M_{o d d}$ and $L$ should be chosen as $2(M-N)+1$ and $\left(M_{\text {odd }}-1\right) / 2=M-N$, respectively. Let $H_{0}\left(e^{j \omega}\right)$ be written as $A\left(e^{j \omega}\right) e^{-2 \omega N}$, where $A\left(e^{j \omega}\right)$ is a complex function but is approximately equal to one if $\beta(z)$ is of sufficiently high order. Together with (2.3), (2.2) can further be simplified to

$$
\begin{gathered}
E(\omega)=e^{-j \omega(2 M+1)}\left(1-\cos (\omega) P(\cos (2 \omega)) \cdot A\left(e^{j \omega}\right)\right), \\
\omega \in[0, \pi / 2] .
\end{gathered}
$$

As $P(\cos (2 \omega))$ is a real-valued function, the minimum value of $|E(\omega)|$ is attained when

$$
\begin{gathered}
P(\cos (2 \omega))=P_{d}(\cos (2 \omega))=\frac{\operatorname{Re}\left(A\left(e^{j \omega}\right)\right)}{\left|A\left(e^{j \omega}\right)\right|^{2} \cos \omega}, \\
\omega \neq \pi / 2 .
\end{gathered}
$$

This is best explained geometrically as shown in Fig. 1(b). $\alpha$ and $A$ stand respectively for $\cos (\omega) P(\cos (2 \omega))$ and $A\left(e^{j \omega}\right)$. It can be seen that $|E(\omega)|$ is minimum when $\alpha A$ is the projection of 1 in the direction of $A$. The corresponding value of $\alpha$ is determined to be $\operatorname{Re}(A) /|A|^{2}$ which leads to (2.5). Writing $x=\cos (2 \omega)$, the design problem becomes a polynomial approximation problem

$$
\begin{aligned}
a_{k, o p t} & =\underset{a_{k}}{\arg \min } \int_{I_{x}} \tilde{W}(x)\left|P(x)-P_{d}(x)\right|^{p} d x, \\
I_{x} & =\left(-1, \tilde{x}_{s}\right] \cup\left[x_{s}, 1\right], \quad \tilde{x}_{s}<x_{s}=\cos \left(2 \omega_{s}\right) .
\end{aligned}
$$

The interval $\left(0, \tilde{x}_{s}\right]$ is an optional disjoint interval used to control the values of $P(\cos (2 \omega))$ in the transition band of $H_{0}\left(e^{j \omega}\right)$ and $\tilde{W}(x)$ is a positive weighting function. If $p=\infty$,

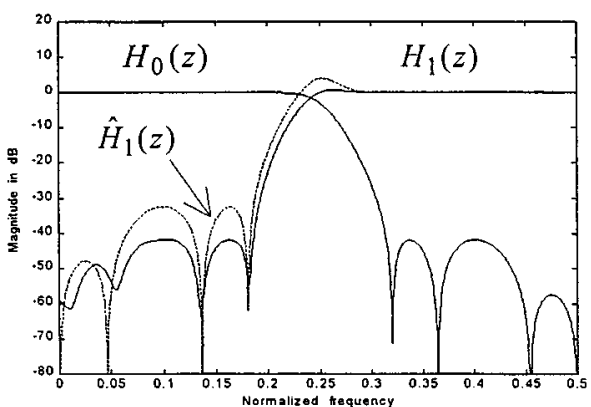

Fig. 2. Frequency responses of example 1. Magnitude responses of $H_{0}(z)$ and $H_{1}(z)$ proposed in this paper (solid lines); proposed $\hat{H}_{1}(z)$ in [2] (dashed line).

(2.6) reduces to the familiar Chebyshev approximation which can be solved using the Remez exchange algorithm with ideal frequency response given by (2.5) and weighting function $\tilde{W}(x)$ given by

$$
\tilde{W}_{\infty}(x)=\left(\cos (0.5 \cdot \arccos (x))\left|A\left(e^{j 0.5 \arccos (x)}\right)\right|^{2}\right) .
$$

Normally, the value of $\tilde{x}_{s}$ is very small and all the alternations appear in the band edges and the interval $\left[x_{s}, 1\right]$. If $p=2,(2.6)$ is simplified to the least square design problem, which again can be solved by the function FIRLS in the signal processing toolbox of MATLAB. We now illustrate the design algorithm using the following examples. It should be noted that the proposed method applies equally well to the design of two-channel linear-phase FIR filter banks, as we shall see later in example 3.

\section{Design EXAmples}

Example 1: To compare the proposed method to that in [2], the lowpass filter in example 1 of [2] is used here. The order of $\beta(z)$ is $N=3 . \alpha(z)$ is designed using the function Remez in the signal processing toolbox of MATLAB. Its order is $M_{\text {odd }}=11$ and the delay parameter $M=8$. Fig. 2 shows the frequency responses of the analysis filters designed by the proposed method (solid lines) and highpass filter $\hat{H}_{1}(z)$ designed by [2] (dashed line). The cutoff frequencies of $H_{1}(z)$ are $\omega_{p}=0.6 \pi$ and $\omega_{s}=0.37 \pi$, which are identical to that reported in [2]. The stopband attenuation, however, is increased to about $40 \mathrm{~dB}$ and the bumping of $H_{1}(z)$ is also significantly suppressed. The system delay on the other hand, is slightly increased from 17 to 23 samples. In this example, the weighting function $\tilde{W}(x)$ is set to one so that the stopband of $H_{1}(z)$ is not equiripple. This shows that the weighting function in (2.7) is useful in achieving an 


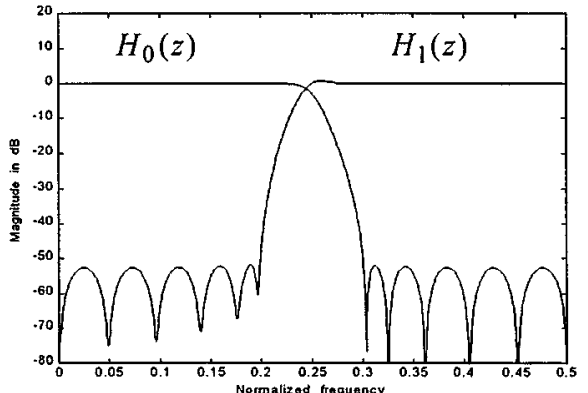

Fig. 3. Magnitude responses of $H_{0}(z)$ and $H_{1}(z)$ in example 2 .

equiripple response in the stopband, as we shall see later in Examples 2 and 3. It can also be seen that by increasing the length of $\alpha(z)$, the stopband attenuation of $H_{1}(z)$ is improved. This demonstrates that the proposed method is able to suppress the bumping in $H_{1}(z)$ and control its stopband attenuation in exchange for a greater system delay.

Example 2: In this example, the proposed method is compared with the general IIR approach proposed in [4]. The orders of $\beta(z)$ and $\alpha(z)$ are chosen as $N=5$ and $M_{\text {odd }}=19$, respectively. The weighting function (2.7) is used to achieve an equiripple response. Fig. 3 shows the frequency responses of the analysis filters. It can be seen that they have a stopband attenuation of approximately $52 \mathrm{~dB}$, which is higher than that reported in [4] $(49 \mathrm{~dB})$ with the same cutoff frequencies. The system delay of the proposed method is also lower than that reported in [4] (39 versus 43). In addition, both the design and implementation complexities of the proposed method are much lower than the general IIR approach in [4].

Example 3: In this example, we compare the proposed method with the maximally flat FIR approach proposed in [6]. In [6], $H_{0}(z)$ and $\alpha(z)$ are chosen as the half-band and maximally flat FIR filters, respectively. As $\alpha(z)$ is not optimized using any measure, its performance is limited. For a fair comparison, $\beta(z)$ is chosen as a type-II FIR function with order $M_{\text {odd }}=15$, which is the same as that in [6]. The order of $\alpha(z)$ is chosen to be 15 . The magnitude response of $H_{1}(z)$ designed by the proposed method is shown as solid line in Fig. 4. The weighting function (2.7) is again used to achieve an equiripple response. The dashed line in Fig. 4 shows the magnitude response of the filter $\hat{H}_{1}(z)$ when $\alpha(z)$ is chosen as a maximally flat FIR function with the same order. It can be seen that the proposed method results in a much sharper cutoff than the maximally flat FIR approach.

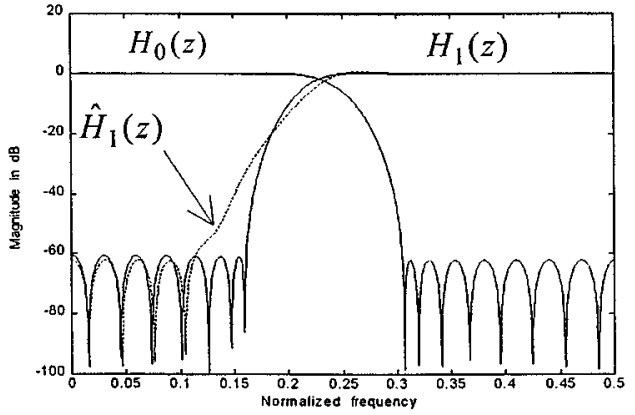

Fig. 4. Magnitude responses of example 3: $H_{0}(z)$ (solid line); optimal $H_{1}(z)$ (solid line); $\hat{H}_{1}(z)$ based on maximally flat $\alpha(z)$ proposed in [7] (dashed line).

\section{CONCLUSION}

A very simple algorithm for designing two-channel causal stable PR IIR filter banks is presented. It is based on a structure previously proposed by Phoong et al. [2]. By using a combination of allpass and linear-phase FIR functions, the bumping problem found in the conventional structural PR filter bank is significantly suppressed. The design problem is formulated as a polynomial approximation problem and is solved effectively using the Remez exchange algorithm. Filter banks with flexible stopband attenuation and system delay can readily be obtained using the proposed algorithm.

\section{ACKNOWLEDGMENT}

The authors would like to thank the anonymous reviewers for carefully reading the manuscript and for their constructive comments, which greatly improved the presentation of this paper.

\section{REFERENCES}

[1] P. P. Vaidyanathan, Multirate Systems and Filter Banks. Englewood Cliffs, NJ: Prentice-Hall, 1992.

[2] S. M. Phoong, C. W. Kim, P. P. Vaidyanathan, and R. Ansari, "A new class of two-channel biothogonal filter banks and wavelet bases," IEEE Trans. Signal Processing, vol. 43, pp. 649-664, Mar. 1995.

[3] T. Q. Nguyen, T. I. Laakso, and R. D. Koilpillai, "Eigenfilter approach for the design of allpass filters approximating a given phase response," IEEE Trans. Signal Processing, vol. 42, pp. 2257-2263, Sept. 1994.

[4] X. Zhang and T. Yoshikawa, "Design of two-channel stable IIR perfect reconstruction filter banks," IEICE Trans. Fundamentals, vol. E81-A, no. 8, pp. 1592-1597, August 1998.

[5] C. W. Kim and R. Ansari, "FIR/IIR exact reconstruction filter bank with application to subband coding of images," Proc. Midwest CAS Symp., vol. 1, pp. 227-230, May 1991.

[6] H. Kiya, M. Yae, and M. Iwahashi, "A linear phase two-channel filter bank allowing perfect reconstruction," Proc. IEEE ISCAS'92, vol. 2, pp. 951-954, May 1992. 